\title{
Review of Asset Pricing Theory and Empirical Research
}

\author{
Zhenzhen Zhao ${ }^{1,2, *}$ \\ ${ }^{1}$ School of Economics and Management, Henan Agricultural University, Zhengzhou, Henan 450046, China \\ ${ }^{2}$ School of Business, The University of Nottingham, Nottingham NG8 1AF, United Kingdom \\ *Zhenzhen Zhao. Email: zhenzzhao@outlook.com
}

\begin{abstract}
This article describes the main asset pricing theories and models and explores the Capital Asset Pricing Model (hereinafter referred to as "CAPM"), the Fama-French three-factor model (hereinafter referred to as "FFTFM"), the Carhart four-factor model (hereinafter referred to as "CFFM"), the Fama-French five-factor model (hereinafter referred to as "FFFFM"), and the Q-factor model (hereinafter referred to as "QFM") in major mature securities markets. The applicability of the above and the applicability in the Chinese stock market, and the applicability of these models in the Chinese stock market are compared, and it is found that these pricing models are not strong in explaining the Chinese stock market. Then combine the characteristics of the domestic market to study the further improvement of the model. Finally, some ideas are put forward for the future research direction of the pricing model.
\end{abstract}

Keywords: Asset Pricing, Empirical Research, Factor Models, China Stock Market

\section{INTRODUCTION}

Asset pricing is the core research content of the modern financial theory, and also one of the areas with rich research results. It has been more than a century since Bachelier started his research in 1900 and now, financial experts have put forward various theories and models. From the single factor model to the multi-factor model, each model has been greatly tested and applied. Financial experts have also found deficiencies and improved them in the test, and continuously researched and developed the most suitable pricing model for the current financial market.

Sharpe (1964) and Lintner (1965) proposed a CAPM based on the "mean-variance" model.CAPM model has become the basic tenet of the financial community, aiming to explain how the securities price is determined according to its own risk or how the balanced price is formed. Since the asset pricing model was proposed, the academic community has made continuous efforts on the effectiveness of the asset pricing model and proposed various pricing models: Fama, French (1993) proposed the FFTFM, which was widely recognized by the academic community and became the research model of the multi-factor Pricing Model. Subsequently, the CFFM, the FFFFM, and the QFM proposed by the academic community are all the mainstream pricing models in the current financial industry. After years of development, China's securities market has gradually grown into an influential capital market. More and more scholars began to pay attention to the ASM, and they have more and more empirical research on the applicability of pricing models in the ASM, to explore the applicability of these models in the ASM and further develop a more suitable pricing model for the ASM on this basis.

\section{LITERATURE REVIEW OF THE THEORIES AND EMPIRICAL RESEARCH RESEARCH RESULTS RELATED TO ASSET PRICING}

\subsection{CAPM Model}

The capital asset pricing model is further developed by Sharp (1964), Lintner (1965), Mossin (1966) on the basis of Markovitz (1952) mean-variance model. It is based on strict assumptions. Because of its simplicity and operability, it has been rapidly developed and widely used abroad since its creation.

my country's research on capital asset pricing models started relatively late. Chinese scholars have conducted a large number of empirical studies on the applicability of the CAPM model to the Chinese stock 
market, and their conclusions are different. Most scholars have reached conclusions after empirical research. To a certain extent, there is a tendency that the CAPM model is not obvious to the effectiveness of my country's capital market. It is precisely because the universality of CAPM has been questioned in empirical analysis, more and more scholars have conducted research on its effectiveness in the ASM.

Zhang Jiaxuan (2017) randomly selected the monthly data of 100 stocks in the Shanghai (hereinafter referred to as "SH") A-share market (hereinafter referred to as "ASM") from 2012 to 2017 for empirical analysis to test the applicability of CAPM and found that the effectiveness of CAPM in China's ASM is not obvious [6]; $\mathrm{Hu}$ Xuejie and Xie Mingyang (2019) to verify the CAPM model with 24 construction and decoration industry stocks in the SH ASM from April 2015 to April 2017 as a sample, it is believed that the CAPM model cannot reflect its pricing function in China's ASM [3]; Sun Xuepeng (2020) Selecting daily data from 2015-2018 of stocks issued over five years in ASM as a sample for empirical research, it is found that the CAPM model is not suitable for China's ASM, but its linear nature is worthy of recognition [4]; Yang Yixuan (2020) selected Shenzhen Stock Exchange listed companies. [5] The monthly data of 10 stocks from 2016 to 2018 are samples for regression analysis. The test results indicate that the applicability of the CAPM model in China's stock market is not well reflected; Zhang Yan and Wang Yideng (2019) are in the effectiveness of the CAPM model. [7] When conducting the research, the securities industry was used as the research object, and the period from 2005 to 2019 was divided into eight bull and bear markets for comparison. The results show that the CAPM model is effective in the securities industry and its applicability in bear markets is higher than in bull markets; Han Zhuolin, Qiao Yuanbo And Shao Xiaoyan (2019) conducted an empirical study and comparison of the effectiveness of the CAPM model on the ASM and the H-share market, and found that the CAPM model is applicable to $\mathrm{H}$ shares, but the effectiveness of ASM is not obvious. [2]

With the development of my country's financial market, more and more scholars have improved and expanded the CAPM model to enhance the applicability of the CAPM model in my country. Cui Jin, Yin Xia, and Huo Qiuju (2020) used listed companies in the ASM as a sample to investigate and found that there is a scale premium in China's capital market, and it is more obvious in small companies, providing a direction for the improvement of the CAPM model, that is Add the scale premium factor to the CAPM model. [1]

\subsection{FFTFM}

Although CAPM has been developed and applied a lot because of its simplicity and ease of operation, its effectiveness, especially in the ASM, has always been controversial. In order to improve the CAPM, Fama and French proposed a FFTFM in 1992, adding excess returns on the market asset portfolio, the scale of the company's market value, and the book-to-market ratio to enhance the interpretation of CAPM. The effectiveness of the later FFTFM in major mature securities markets has also been supported by scholars' empirical research.

Whether the FFTFM is applicable to the ASM has also become a hot spot for scholars from all walks of life. Lu Yuyan (2015) selected all stocks in the Chinese ASM from January 2012 to December 2012 as samples for empirical research and testing. Judging from the results, the applicability of the FFTFM in the ASM is not as effective as the mature securities markets of advanced economies can play its role, and its effectiveness needs to be further explored; Zeng Hui (2016) compared the period from May 2009 to 2013 The weekly trading data of all stocks in China's ASM in April is a sample for empirical analysis. It is believed that the FFTFM has a strong ability to explain China's ASM. The two scholars conducted research on the same stock market but came to different conclusions. The reason is that the time range selected by Zeng Hui includes the period of my country's financial crisis. In the time range she studied, there are both bear and bull markets. The inspection range is longer and the sample types are more abundant. Liu Hui (2019) uses the monthly data of China's ASM from April 2014 to March 2017 as a sample to conduct empirical analysis, confirming that market premium factors, company market value scale factors, and book-to-market value ratio factors all contribute to the monthly return rate of stocks We have reason to believe that the FFTFM is effective for China's ASM; in 2019, Meiqi Hu and Lin Fengyan's analysis of the FFTFM for China's 22 green funds (green funds are only for energy-saving and emission-reduction strategies. The special investment fund established for the development of the carbon economy and environmental optimization and transformation projects aims to promote the development of energy conservation and emission reduction through capital investment. [8] The results show that the FFTFM is effective for China's green fund market.

In order to make the FFTFM more suitable for my country's stock market, many scholars have improved the FFTFM and added new factors for further research to test whether the expanded FFTFM can have a stronger explanatory power for the ASM [9]. Yin Liya (2018) added investor sentiment index as a sentiment factor to the FFTFM, and conducted empirical research using monthly data from April 2010 to March 2016 as a sample to explore whether the expanded FFTFM is effective for my country The market has stronger applicability, and the results show that the FFTFM after 
adding the sentiment factor can have a better explanation for the rate of return. [10] In 2017, Zhang Bing and Chen Xiaoying studied the effect of lowpriced stocks in my country, and replaced the book value ratio with the nominal stock price factor [11]. Using all ASM stocks from December 1999 to December 2015 as the research sample, they found new three factors empirically the predictive power of the model is better than the original FFTFM.

\subsection{CFFM}

Scholars have never stopped their research on the factors affecting asset pricing, and some breakthroughs have been made. In their research on mature securities markets in developed economies, they have discovered momentum effects in these markets (Jegadeesh and Titman, 1993). Afterwards, Carhart extracted the momentum factor from the momentum effect and proposed a CFFM in 1997.

The applicability of the CFFM in the ASM has also attracted the attention of a large number of scholars. Ouyang Zhigang and Li Fei (2016) selected monthly SH and Shenzhen (hereinafter referred to as "SZ") ASM trading data from January 1997 to June 2015 (222 months in total) as samples, and conducted a study on the applicability of the CFFM in the Chinese ASM. In empirical research, the author found that the CFFM is suitable for the Chinese stock market, and believes that the CFFM has stronger explanatory power than the CAPM and FFTFM. However, Cao Binjie's (2019) empirical research gave a different view. He used the data of the SH Stock Exchange from July 2005 to June 2018 as a sample to study the explanatory power of the CFFM on the Chinese stock market and compare it with the explanatory power of the FFTFM is compared. The empirical results show that the CFFM is suitable for the ASM. However, through regression analysis, it is found that the regression result of the momentum factor is not significant, that is, the momentum factor cannot explain more of the excess that the FFTFM fails to explain. The rate of return, in other words, the solution of the CFFM is that the strength is not better than that of the FFTFM.

\subsection{FFFFM}

Although the FFTFM and the CFFM are widely recognized and used by the academic and practical circles, with the research and empirical research in the academic circles, it has been discovered that new anomalies cannot be explained by the FFTFM and the CFFM. The most important is the profit anomaly and the investment anomaly. In 2015, Fama and French added profitability and investment style factors to the FFTFM, and proposed a FFFFM on the basis of the FFTFM to enhance the explanatory power of the model.
The applicability of the FFFFM in the international market has been tested, which is of great significance to the empirical test of the applicability of the FFFFM in the ASM. However, the current Chinese academic circles have not paid enough attention to the use and testing of the FFFFM. Only a few scholars have tested the applicability of the FFFFM in the ASM, and their conclusions are not consistent. Zhao Shengmin, Yan Honglei and Zhang Kai (2016) selected monthly data of all stocks in China's ASM from January 1993 to December 2014 as a sample to conduct an empirical study on the applicability of the FFFFM in the ASM, and found that the FFFFM is in China The applicability of the FFFFM is not as good as the FFTFM [17]; Sun Ce, Jiang Xuning, and Huang Heliang (2019) take monthly data of listed companies in the ASM from 2000 to 2018 as a sample to conduct an empirical study on the applicability of the FFFFM in the Chinese stock market [18]. It is found that although the FFFFM is not perfect, it has better explanatory power than other pricing models. Li Zhibing, Yang Guangyi, Feng Yongchang, and Jing Liang (2017) used monthly data of listed company stocks in China's ASM from July 1994 to August 2015 as samples to test the applicability of the FFFFM in the ASM and found five factor model has stronger explanatory power than the CAPM, FFTFM, and CFFM. The author also uses the equity split reform as a demarcation point to explore whether the capital market efficiency has improved before and after the share reform. The applicability of the market is better than before the share reform. Gao Ke and Zhang Linzhengzi (2017) randomly selected a total of 10 stocks from different industries in the China ASM, using the data of these stocks from January 2008 to June 2015 as samples, and divided them into bear market, shock market and bull market In the three stages, the FFFFM is empirically tested for the applicability of the ASM [19], and it is found that the FFFFM is not strong in the explanation of the bull market, and its applicability in the bear and shock markets is not better than the FFTFM. [20]

In order to enhance the applicability of the FFFFM in the ASM, some scholars have tried to improve the FFFFM. For example, Sun Ce, Jiang Xuning and Huang Heliang (2019) reconstructed and improved the investment factors in the FFFFM based on the characteristics of the ASM, and strengthened the interpretation of the FFFFM. [21]

\section{$2.5 Q F M$}

In order to explain the anomaly in the stock market more effectively, Hou, Xue, and Zhang (2015) proposed the QFM based on the investment pricing theory formed by Tobin's Q theory, which contains Four factors including market factor, scale factor, investment factor and profit factor are analyzed. [22] Through their 
empirical research, they found that, compared to the Fama-French three-factor pricing model and the Carhart four-factor pricing model, the Q-factor pricing model is more effective in explaining the anomaly. [23]

Hou, Xue, and Zhang (2015) compared the FamaFrench five-factor pricing model and the Q-factor pricing model's ability to explain 74 market anomalies. They believe that the four factors of the Q-factor pricing model can fully explain the factors in the FFFFM, especially the momentum and profit anomalies that exist in the market.

Scholars at home and abroad have concentrated on the capital asset pricing model and the Fama-French three-factor pricing model and the Fama-French fivefactor pricing model in the multi-factor pricing model, and there is not much research on the Q-factor pricing model. However, Hou, Xue and Zhang have conducted many empirical studies to prove that in the single-factor market anomaly, the Q-factor pricing model performs better than the models mentioned above, so this model has the value of in-depth research. Bi Shaogang (2015) studied the applicability of the QFM in China's ASM and found that the QFM is better than Fama-French in explaining the stock market anomalies such as asset growth effects, earnings announcement effects, and comprehensive issuance effects. FFTFM, and its performance in the SZ Stock Exchange is significantly better than that in the SH Stock Exchange, and the two are on par. Fang Yi, Meng Jixian, and Qu Junxue (2019) tested the Q-factor pricing model's ability to explain market anomalies. The empirical research results show that in the interpretation of market anomalies, the QFM and Fama-French five factors Each model has its own merits. However, in general, the QFM is better than the FFTFM and the FFFFM in explaining certain market anomalies: the effect of the $\beta$ value, the effect of comprehensive stock issuance, the effect of accrued profits, and the momentum The explanatory power of the effect, the $\mathrm{B} / \mathrm{M}$ effect, and the investment effect is stronger than the FFTFM; the QFM performs better in the four market anomalies: volatility effect, accrued profit effect, $\mathrm{B} / \mathrm{M}$ effect, and investment effect. Better than the FFFFM.

Hou, Mo, Xue and Zhang (2020) found in their research that the expected growth factor is a factor that has a greater impact on expected returns but is neglected. They added the expected growth factor to the QFM and constructed a q5 factor model. The model was tested and found that the q5 factor model performed better than other pricing models.

\subsection{Comparative Analysis of Mainstream Models}

Capital asset pricing model, FFTFM, CFFM, FFFFM, and QFM, as the mainstream models in the current financial market, have always been hot topics of concern and discussion in the financial community. Financial scholars have never stopped empirical research on the applicability of these models in the Chinese stock market. Scholars have tried to find a pricing model that is suitable for the Chinese stock market. The capital asset pricing model is the backbone of modern financial market price theory. This model is widely used in the financial field because of its simplicity and easy calculation. However, the capital asset pricing model also has shortcomings that cannot be ignored. On the one hand, it is based on strict assumptions and the assumptions are difficult to achieve; on the other hand, the value in the capital asset pricing model is difficult to determine, which makes the CAPM model controversial. Regarding whether CAPM is applicable to the Chinese stock market, scholars have different opinions. The applicability of the FFTFM based on the CAPM in the ASM has also been concerned by scholars from all walks of life. After a lot of empirical analysis, scholars have not reached a unified opinion on the effectiveness of the FFTFM in the ASM, but the FFTFM the overall performance is better than that of the CAPM model. Later, the momentum factor was introduced on the basis of the FFTFM to form a CFFM, which was compared with

The FFTFM and CAPM have higher explanatory power. In the research on the applicability of the FFFFM in the Chinese stock market, scholars cannot find evidence that the FFFFM is better than the FFTFM. In the research on the QFM, scholars have found through empirical research that the QFM performs better than the three-factor and CFFMs in the ASM, and can also explain and supplement the factors in the FFFFM. On the whole, although the multi-factor model has certain advantages over the CAPM model and has better applicability to the ASM, it is not obvious enough. The mainstream model also needs to be further improved.

\section{CONCLUSION}

In the academic research on the applicability of the CAPM model in the ASM, it is found that the CAPM model is not effective in the ASM. Scholars also try to add a scale premium factor to the CAPM model to improve the interpretation of the CAPM model in the ASM; In empirical research, scholars found that the effectiveness of the FFTFM in major mature securities markets is worthy of recognition. The FFTFM has certain effects on the ASM, but its effectiveness is not as stable as its effectiveness in mature markets. Chinese scholars also added the sentiment factor to the FFTFM and replaced the book value ratio with the nominal stock price factor. They found that the improved FFTFM strengthened the interpretation of the ASM; In the research on the applicability of the CFFM in the ASM, scholars have given different opinions. Whether the 
applicability of the CFFM in the ASM is better than the FFTFM requires further research; for the FFFFM in China Whether the applicability of the market is better than the FFTFM is also where scholars are divided; although domestic and foreign studies on the QFM are not as good as the FFTFM and the FFFFM, it is empirically found that the QFM has an overall anomaly The explanation power is better than the FFTFM and the FFFFM, which has the value of in-depth research.

\section{FUTURE RESEARCH OUTLOOK}

The research on asset pricing in academia has always been the core, and asset pricing theories and models have been developing and improving. For example, behavioral finance theories developed from the perspective of psychology have added emotional factors to the FFTFM and combined with the ASM's Features The reconstruction and improvement of the investment factors in the FFFFM, and the addition of expected factors to the QFM are all directions that are worthy of further research. In addition to the application of some portfolio management strategies such as Frazzini and Pedersen, LH (2014) present a model with leverage and margin constraints that vary across investors and time. By controlling the batting against the beta $(\mathrm{BAB})$ factor, We can increase the excess return [25]. And Moreira and Muir (2017) propose the volatility management portfolio strategy to improve the return of investors. Asset pricing has many directions worthy of further research, and we need to explore further. [24]

\section{REFERENCES}

[1] Cui Jin, Yin Xia, Huo Qiuju. Research on the Improvement of CAPM Model in China's Capital Market-An Empirical Analysis Based on Scale Premium[J]. China Assets Appraisal, 2020 (05).

[2] Han Zhuolin, Qiao Yuanbo, Shao Xiaoyan. Analysis of the effectiveness of CAPM series models-a comparative analysis based on the industry data of $\mathrm{SH}, \mathrm{SZ}$ and Hong Kong stock markets[J]. Investment Research, 2019(10).

[3] Hu Xuejie, Xie Mingyang. Research on the application of capital asset pricing model based on data from China's ASM [J]. Economic Perspective, 2019(5).

[4] Sun Xuepeng. Practical analysis of capital asset pricing model theory $[\mathrm{J}]$. Finance and Economics, 2019(02).

[5] Yang Yixuan. An Empirical Test of the Applicability of the CAPM Model in the SZ Securities Market[J]. Modern Commercial Industry, 2020 (23).
[6] Zhang Jiaxuan. Empirical analysis of CAPM model in SH ASM[J]. China Market, 2017(20).

[7] Zhang Yan, Wang Yideng. Research on the effectiveness of the CAPM model-based on the empirical comparison of China's securities industry in the bull and bear market $[\mathrm{J}]$. Foreign Economic Relations and Trade, 2019 (05).

[8] $\mathrm{Hu}$ Meiqi, Lin Fengyan. Research on the applicability of the FFTFM to my country's green funds[J]. Times Finance, 2019.

[9] Liu Hui. Empirical analysis of China's stock market based on the FFTFM [J]. Market Weekly, 2019, (08).

[10] Yin Liya. An Empirical Study on the Impact of Investor Sentiment on Stock Returns-Based on the FFTFM[J]. Investment and Finance, 2018, (06).

[11] Lu Yuyan. Analysis of the applicability of the FFTFM in the Chinese stock market[J]. Wuhan Finance, 2015, (02).

[12] Zhang Bing, Chen Xiaoying. Research on the effect of low-priced stocks in China's stock market-based on the FFTFM test[J]. Financial Forum, 2017, (10).

[13] Zeng Hui. Empirical analysis of CAPM and its derivative models in $\mathrm{SH} \mathrm{ASM}[\mathrm{J}]$. Financial Perspective, 2016, (01).

[14] Cao Binjie. An Empirical Study on the CFFM of China's Stock Market[J]. Economic Aspects, 2019, (14).

[15]Ouyang Zhigang, Li Fei. Research on the applicability of the four-factor asset pricing model in China's stock market[J]. Research in Financial Economics, 2016, (02).

[16] Xia Xiaolong, Mao Yue. A review of multi-factor asset pricing theory [J]. Think Tank Times, 2020, (04).

[17] Fang Yi, Meng Jixian and Qu Junxue. A comparative study of multi-factor pricing models based on market anomalies[J]. Quantitative Economics Research, 2019, (01).

[18] Kewei Hou, Chen Xue, Lu Zhang, Digesting Anomalies: An Investment Approach, The Review of Financial Studies, Volume 28, Issue 3, March 2015, Pages 650-705.

[19] Kewei Hou, Haitao Mo, Chen Xue, Lu Zhang, An Augmented QFM with Expected Growth, Review of Finance, rfaa004, https://doi.org/10.1093/rof/rfaa004

[20] Hou, Kewei and Xue, Chen and Zhang, Lu, A Comparison of New Factor 2015-03-05, HKUST 
Finance Symposium 2016: Active Investing and Arbitrage CModels (April 18, 2017). Fisher College of Business Working Paper No . apital, Charles A. Dice Center Working Paper No. 201505, Available at SSRN:

https://ssrn.com/abstract=2520929 or http://dx.doi.org/10.2139/ssrn.2520929

[21] Shao-Ding Liu, Zhi-Xing Wang, Wen-Jie Wang, Jing-Dong Chen, and Zhi-Hui Chen, "High Qfactor with the excitation of anapole modes in dielectric split nanodisk arrays," Opt. Express 25, 22375-22387 (2017)

[22] Zhao Shengmin, Yan Honglei and Zhang Kai. Is the FFFFM better than the FFTFM? Empirical evidence from China's ASM[J].Nankai Economic Research,2016,(02).

[23] Shen Yifeng. The FFFFM of capital asset pricing: evolution and future research directions[J]. Financial Research, 2015, (06).

[24] Li Zhibing, Yang Guangyi, Feng Yongchang and Jing Liang. Empirical test of FFFFM in Chinese stock market[J]. Financial Research, 2017, (06).

[25] Gao Ke, Zhang Linzhengzi. Investor sentiment, FFFFM and portfolio returns[J].

[26] Sun Ce, Jiang Xuning and Huang Heliang. Research on the Validity Test and Improvement of the Fama FFFFM in the Chinese Securities Market[J]. Journal of Wuyi University, 2019, (03).

[27] Cao Liyan. Comparison of China's market efficiency between Marfa's FFFFM and FFTFM[J]. Modern Economic Information, 2017, (01).

[28] Yang Qilin, Yang Jing and Lu Longchao. Comparison of Fama-French multi-factor model and CAPM model-evidence from the ASM [J]. SME Management and Technology, 2020, (06).

[29] Moreira, A. and Muir, T. (2017), VolatilityManaged Portfolios. The Journal of Finance, 72: 1611-1644.

[30] Frazzini, A. and Pedersen, L. H. (2014). Betting against beta. Journal of Financial Economics, 111(1):1 - 25 . 